\title{
Explaining a Scientific Concept for Page and Screens
}

\author{
Michael Ennis, Regis University
}

January 1, 2017

\begin{abstract}
While students learn valuable skills by composing multimodal works, these assignments can also help students master traditional writing genres by defamiliarizing some of the "design choices" they make when writing. Requiring students to revise a traditional written essay into a video accomplishes two key goals in both lower level and advanced writing classes. It updates writing curricula to provide students experience with the kind of writing they will do in other classes. Furthermore, reflecting on the revision process enhances student appreciation for the importance of clear prose, careful exposition, and logical organization.
\end{abstract}

In the summer of 2014, the University of Colorado-Boulder's Program for Writing and Rhetoric (PWR) began to retool its curriculum by providing faculty training in digital composition. The PWR's curriculum was designed for students in the university's colleges of business, arts and sciences, and engineering, and the digital composition initiative prepared the PWR to contribute to Boulder's new College of Media, Communication and Information. Also, the PWR standardized its learning outcomes, ensuring students learned to compose and critically analyze digital texts. The Center for Digital Storytelling (www.storycenter.org) in Denver facilitated the training, taking a hands-on approach. During the one-week training, PWR faculty created five-minute videos that told a personal narrative, the specialty of the Center. While the workshop accomplished several key goals - providing participants with technical skills, bringing our department together, demystifying the process for skeptics, and being fun-it did focus on a relatively narrow mode of digital communication: personal narrative.

Since I was slotted to teach upper-level classes in science writing and business writing in the fall, I was concerned that the digital work would not transfer to the learning goals of those courses. While it is possible to imagine productive personal narratives in courses with a technical focus, I feared that the time and effort students put into 
learning the technology would have diminished returns if not applied to a genre currently more central to those fields. A second concern sprang out of many of our workshop's participants: video was too constrictive, and students should have the option of which digital medium to compose in. Giving students liberty to choose a video or infographic or podcast is a common way to approach these assignments. While I saw the benefit in giving students that freedom, I wanted to also recreate the focused workshop atmosphere that the Center for Digital Storytelling had brought to our department. The fact that we all struggled with similar technical issues facilitated a sense of accomplishment and collaboration. As Lunsford (2006) pointed out when discussing the implementation of similar programmatic changes at Stanford University, we must always be careful to balance technical, multi-modal writing with instruction in rhetoric and basic writing. Teaching this assignment reminded me that the two should not be seen as separate. When properly integrated into an assignment sequence, a multimodal project can reinforce students' writing for the page and provides an alternative to the traditional capstone sequence of revising a research paper into a presentation.

One fundamental existing assignment in my upper level science course called upon students to write a concise explanation of a recent scientific discovery or challenging scientific concept to an audience of non-experts. (I used a similar assignment sequence in my business writing course.) I designed a multimedia revision and extension of this project, in which students would revise their essay into a video. Later in the semester, I gave students freedom to choose their genre, but for this early unit I insisted on digital video. After reading several examples of science writers explaining concepts and several "how to" readings about science writing, students put some of the moves into practice (see step one of the assignment). We discussed several of the techniques writers used to engage the audience, such as using striking facts; profiling scientists; incorporating images; emphasizing the "so what?"; and building the explanation in stages. Students then tried to use some of these techniques in their written explanations.

For the next stage in the assignment, students used the written explanation as the basis for a short video. By far the biggest challenge in this phase was getting students up to speed with video editing software. I selected WeVideo-a cloud-based video editing platform - for three reasons: it is free (or very inexpensive for an upgraded one-month subscription); relatively simple to use; and, being cloud-based, available from any computer with a Flash-enabled browser. I used a video explanation I had created in WeVideo as a model, which allowed me to show them what the video editor looked like. This greatly demystified the process.

More important than the technical aspects of putting the video together, the rhetorical choices students made comprised a significant portion of our discussions. In addition to my own video, we screened several model video explanations - all of which took different approaches to engaging their audiences. One, for example, showed time-lapsed video of the transformations wrought by the reintroduction of wolves into Yellowstone (Sustainable Human, 2014). Another animated a graph showing perceptions of income distribution in the United States (Politizane, 2012). My own video used voiceover, text, 
still images, and embedded slide and text elements to explain the narrative on a Maya vase. The resulting conversations stimulated much debate and critical thinking about which aspects played the most crucial role in not only entertaining viewers, but also clearly explaining ideas. We also discussed audience. Their written assignments were clearly framed as popular science writing. Students needed to consider the intended audience for the videos. Students ultimately created videos using a variety of approaches, but most tended towards using a voice to explain a series of images. A few of the braver ones experimented with talking headshots and video collages.

I provided students with time to work on their videos in class, which enabled some of my most lively and collaborative workshop experiences. It was satisfying to see students struggle through mastering certain technical skills with a tenacity they did not often give to the written word. These struggles included simple but unfamiliar aspects of editing, like synching the voiceover to the appropriate image or video clip. At a more sophisticated level, students began to ponder what was strictly necessary. One student adopted the mantra "What does it add?" and applied it ruthlessly to music soundtracks and redundant verbal explanations of images. As a capstone to the project, students analyzed their own work in a writer's statement.

The project produced several excellent results. At the broadest level, producing a video defamiliarized the writing process. In their reflections, students commented on how they had taken their writing choices for granted, and creating the video prompted them to consider those design choices more carefully. While making the video, we discussed design choices at length, which enabled students, through the reflection, to question the "design choices" in their writing. Arbitrary breaks and clunky transitions stood out on the screen more boldly than awkward paragraphs and serial organization did on the page. I think this results from two related tendencies in how we teach writing. First, the opportunity cost for superfluous words in a paper is low for students: in fact, they are often rewarded for exceeding page limits. However, adding voice and images in a video editor takes time and effort to get right. They know when a video is not properly synched and looks sloppy, and they value doing it correctly. Second, students have written many papers and often take for granted fundamental "moves," such as using a paragraph as an organizational choice. Creating video transitions for the first time impressed upon them the importance of carefully planning their written organization.

Better still, the video forced students to reconsider voice and image in their prose. While we discussed how professional writers incorporated images, only one or two students followed suit when they wrote their essays. Many noted that missed opportunity. And while I often urge students to read their prose aloud, I know that few do. However, the video forced them to listen to their prose, especially since many simply tried to use an unrevised essay as a script, which they soon realized would not work. Hearing the awkward and sometimes convoluted sentences issue from computer speakers made students rethink their prose voice more thoroughly than I had seen students do in versions of the course that did not include this assignment.

Not everything worked as planned. The pacing of my original assignment did not offer 
enough time for students to integrate their skills. The timeline reflected in the attached prompt was extended about a week beyond my original plan. My students' wildly different beginning skillsets also presented a challenge. Some students completed their videos with relative ease. In one section this worked well: they helped their classmates during workshops. In another, it led to unrest. Finally, WeVideo proved divisive. Some students enjoyed its convenience, ease-of-use, and affordability. Others complained that it offered fewer features than iMovie, for example. But iMovie requires students to own a Mac. Students were unanimous in one criticism of WeVideo: its performance slowed considerably on residential Internet compared to the campus Wi-Fi. When I do this assignment again, I will experiment with allowing students to choose their platform.

I have often asked students to revise their writing into another genre, most commonly by having them do a presentation. However, having a focused, challenging, digital genre to collaborate on not only taught students valuable skills working in different media, but also made them better composers of prose. My experience using the science writing assignment sequence in a business writing class to equal success suggests that this sequence of assignments can be adapted for writing intensive courses across the curriculum.

\section{Assignment: Explaining a Scientific Concept For Page and Screens}

See the Supplementary Files for this article at thepromptjournal.com for a PDF facsimile of the original formatting of this assignment.

\section{Project One: Scientific Writing}

\section{Timeline}

- Week One: In-class Workshop

- Week Two: Final Draft Due

Your first task will be to explain a difficult scientific concept or recent discovery in writing for an audience of non-specialists. You can choose anything, so long as it is a sufficiently complicated idea to require three pages, at least, to explain. You will have seen and discussed several examples of explanation so far: the importance of micro flora (Buhler), the microbiology of human evolution (Kolbert), the development of AI (Mueller), etc. We have also looked at a couple of explanations about how to explain a scientific idea to non-specialists.

In particular, you should work on the skill - discussed in detail by science writers George Johnson and Joshua Schimel - of developing the explanation in stages. Start with a schema or analogy and then build on it to help a non-expert reader through the idea step-by-step. 
In addition to this essential skill, you will develop and practice several moves essential for science writing:

a) Put the explanation into the context of a brief narrative - this can be a profile or even a narrative or your own discovery.

b) Work on providing definitions and glosses that are concise but complete.

c) Give background on the problem or discovery. Why is it significant? How did it change our thinking about a given problem or phenomenon?

d) Use a striking fact, image, or statistic to generate interest in the explanation.

Finally, no matter which techniques you use, your essay should also explain the significance of the concept. What can readers understand now that they "get" the idea? How have you walked them from data to information to knowledge to, finally, understanding?

\section{Submission Details:}

- Bring a laptop or tablet to class on Monday (Week 1) to use for the workshop. Be prepared to email the draft to your workshop group.

- Submit to Desire 2 Learn (D2L) assessments dropbox using the link with the appropriate title.

- Upload a .doc or .docx file.

- Minimum 900 words

- Use a standard 12pt. font (e.g. Times New Roman, Cambria).

- Include references at the end.

- You may use graphics, although do not rely exclusively on them.

\section{Project Two: Digital Video}

\section{Timeline}

- Week Three: Bring draft of script and images for storyboarding. Setup WeVideo account and watch tutorials.

- Week Three: In-class work in WeVideo. Bring a copy of your audio file in mp3 format.

- Week Four: Bring a rough cut for workshop and feedback.

- Week Five: Publish project and post link to D2L.

You will revise your explanation into a three-minute video. This assignment will help you as science writers in three key ways:

a) You will develop skills working with digital media. 
b) You will gain skills using images to enhance your explanations, making them central to your writing.

c) You will continue to develop your awareness of the demands of working in different media and genres for different audiences.

\section{Planning}

Over the weekend, you should look carefully at your explanation essay. Think about which parts might serve as useful voiceovers in a video, and which parts you need to cut. Consider how you might best reorganize your explanation given the possibilities and limitations of working in a digital medium. Consider the strategies from the videos we screened in class and think which approaches are best suited for your explanation.

Read your essay out loud several times. Revise for clarity as a spoken-word piece. Time it to determine if you need to cut or expand. Also, note what kind of images you need to accompany the different parts of the video. Are there parts that might not have any accompanying images or video? You will probably need to revise so that the images keep moving and your audience stays engaged. You will also need to consider issues of redundancy. What you explained clearly (and at length) in writing might be easily captured in one image.

For Monday (Week 3), bring in a revised version of the essay that can serve as the basis of the "script." You should also begin collecting images, music, video clips, etc. that you will use in the video. Ideally, images should be 200dpi and clips HD. Audio should be in mp3 format. In class, we will go over storyboarding techniques and I will show you some of WeVideo's features. You will have a chance to start storyboarding in class.

Between Monday and Wednesday (Week 3), you should record your voiceover. While WeVideo has a record option, being cloud-based it does not always provide the best quality. Using Audacity (an open-source audio editing program) will provide better audio. I will cover how to use it in class.

On Wednesday (Week 3), you will have time to construct your video in class. I expect this to be a collaborative process. You can use each other as resources to figure out how to overcome some of the technical hurdles.

By next Monday (Week 4) you will have completed a rough cut of the video. You will receive feedback in small-group workshops on the clarity of the explanation and the visual style. You will have a chance during the second half of class to revise, ask questions, and figure out technical fixes. On Wednesday (Week 4) you will have time in class to work on the video and get help from your classmates and me.

Publish your video the following Monday (Week 5). Do not do it until you are certain it is final. I will show you how to publish it in class. You should then post the WeVideo link to D2L dropbox. This has to be done by $2 \mathrm{pm}$ so that I can create a playlist for screening in class. We will watch everyone's videos so that you can see multiple approaches to multimedia explanation. 


\section{Submission Details:}

- Post your WeVideo link to D2L by Monday.

- Aim for the video to be around three minutes. (No shorter than 2:30, no longer than 4:00)

- You'll have the chance to reflect on what worked well, what didn't, and what you might have done differently if you had more technical skills in the end-of-unit reflection.

\section{Project Three: Writer's Statement}

\section{Timeline}

- Week Five: Screen Videos in Class

- Week Five: Post Writer's Statement to D2L

As a capstone to the first unit, write a 750-word writer's statement that discusses both assignments and analyzes the uses and limitations of the different media. Specifically, comment on all of the following:

1. Which media (digital video vs. written essay) did you think helped you explain your topic best? Why? What are the affordances and limitations of each? How might you use the affordances in one medium to augment the other?

2. If you were to revise your essay based on your experience working in digital media, what would you change? Be as specific and concrete as possible. Consider: How would you incorporate images? How would you revise your prose? What would you cut? Add?

3. Describe the strengths and weaknesses of your video. Which aspect(s) explains your topic most effectively? What would you have done in your multimedia had you more time or technical expertise? Discuss three of the videos we watched together in class: What might you learn from them about different strategies in the media?

\section{Submission Details}

- Submit the reflection to D2L using the link with the appropriate title.

- 750-word minimum. 


\section{Grading}

In general, the grades for each part of this assignment will reflect how well your finished product employs strategies from our common readings and videos. I will allot time in class to collaborate on constructing a detailed rubric. As you work on the video, you might face some technical hurdles. While the final draft should be polished and mostly glitch-free, you'll have a chance to reflect on the challenges of working in the digital medium in your writer's statement. It will be more important to reflect critically on your rhetorical choices than to master all of the technical aspects of making the video.

\section{References}

Buhler, B. (2011). The Teeming Metropolis of You. California Magazine. Retrieved from http://alumni.berkeley.edu/california-magazine/fall-2011-good-fight/ teeming-metropolis-you

Johnson, G. (2005). Explanatory Writing. In D. Blum, M. Knudson, \& R. M. Henig (Eds.), A Field Guide for Science Writers: The Official Guide of the National Association of Science Writers (2nd edition, pp. 132-137). Oxford; New York: Oxford University Press.

Kolbert, E. (2011). Sleeping with the Enemy. The New Yorker, 87(24). Retrieved from http://www.newyorker.com/magazine/2011/08/15/sleeping-with-the-enemy

Lunsford, A. A. (2006). Writing, technologies, and the fifth canon. Computers and Composition, 23(2), 169-177. http://doi.org/10.1016/j.compcom.2006.02.002

Mueller, T. (2010). Your Move. In J. Cohen (Ed.), The Best of the Best of American Science Writing (pp. 278-294). New York: Harper-Collins.

Politizane. (2012, November). Wealth Inequality in America. Retrieved from https: //www.youtube.com/watch?v=QPKKQnijnsM

Schimel, J. (2011). Writing Science: How to Write Papers That Get Cited and Proposals That Get Funded (1 edition). Oxford; New York: Oxford University Press.

Sustainable Human. (2014, February). How Wolves Change Rivers. Retrieved from https://www.youtube.com/watch?v=ysa5OBhXz-Q 